Rev. Biol. Trop., 47(1-2): 239-243, 1998

www.ucr.ac.cr www.ots.ac.cr www.ots.duke.edu

\title{
Conducta alimentaria de cuatro especies de aves playeras (Charadriiformes: Scolopacidae) en Chametla, B.C.S., México
}

\author{
Georgina Brabata ${ }^{1-2} \&$ Roberto Carmona ${ }^{1}$ \\ 1 Departamento de Biología Marina. Universidad Autónoma de Baja California Sur. Apartado Postal 19-B. La Paz, \\ Baja California Sur, México. \\ 2 Centro Interdisciplinario de Ciencias Marinas. Av. Politécnico s/n. La Paz, Baja California Sur, México ${ }^{1-2}$ FAX (112) \\ 1-28-0119brabata@calafia.uabcs.mx
}

Recibido 16-IV-1998. Corregido 3-XII-1998. Corregido 9-XII-1998

\begin{abstract}
Feeding behaviour of four species for large shorebirds (Scolopacidae) was studied in a tidal mudflat, Chametla, Mexico, to determine the foraging efficiency of Limosa fedoa, Numenius phaeopus, Numenius americanus and Catoptrophorus semipalmatus in a stopover/wintering area; and their activity with the tide cicle. The study area was visited in the winter of 1993 during the daylight hours. Each species was observed for $15 \mathrm{~min}$ during each tide type. The tide type does not affect the frequency with which the species use the different foraging strategies. In general, probing was more efficient than pecking. Considering distribution and prey, L. fedoa had the highest efficiency with this feeding strategy. Both species of Numenius were more efficient with pecking than other species. C. semipalmatus has the highest efficiency because it is an oportunistic species, L. fedoa is generalistic, both Numenius are especialists and C. semipalmatus is oportunistic.
\end{abstract}

Key words: Feeding behaviour, efficiency, foraging strategies, Baja California Peninsula, large shorebirds.

En la porción norte del Continente Americano se ubican las áreas de reproducción de al menos 42 especies de aves playeras. Una característica generalizada de estas especies es la migración, por lo que permanecen durante el invierno en zonas sureñas, con condiciones menos extremas. Estas migraciones, dependiendo de la especie, pueden implicar áreas de invernación tan norteñas como California, o tan sureñas como Argentina (Paulson 1993). Durante su migración estas aves utilizan una serie de sitios eslabonados (aves de paso), por lo que la desaparición de uno de ellos puede ocasionar un efecto negativo acumulativo muy pronunciado. Buena parte de las áreas de paso y algunas de las de invernación se encuentran en los trópicos y subtrópicos, y pese a que estas áreas son tan importantes como las de reproducción se han realizado pocos estudios en ellas (Brabata 1995).

Algunos autores plantean la posibilidad de que exista una relación entre el tamaño del ave y el sustrato de las playas que frecuenta (Baker 1977, Brabata 1995). Así, las especies grandes prefieren playas menos fangosas que las elegidas generalmente por playeros más pequeños. Dado que el método de captura y la presa están correlacionados, de existir diferencias en los métodos, éstas se pueden interpretar como bajas probabilidades de competencia. En este contexto, el trabajo presente pretende determinar la eficiencia de los diferentes métodos de 
búsqueda y captura de presas por cuatro especies de escolopácidos grandes (Limosa fedoa, Numenius phaeopus, N. americanus y Catoptrophorus semipalmatus), en un área de paso e invernación y su relación con el tipo de marea.

El trabajo se realizó en la Ensenada de La Paz, una laguna somera con profundidad máxima de 10 m, comunicada con la Bahía de La Paz por un canal de un kilómetro de ancho. El litoral de la ensenada es arenoso con algunas zonas fangosas. El clima de la región es seco y desértico, no cuenta con aportes constantes de agua dulce, sólo los proporcionados por las lluvias estacionales cuyo promedio anual fluctúa entre 180 y 250 mm (Espinoza 1977). Al sur de la Ensenada de La Paz se localiza la playa de Chametla (2406'23.7'N, 110²4'51.0'W), a $10 \mathrm{~km}$ de la ciudad de La Paz. Tiene una superficie de $1.43 \mathrm{~km}^{2}$ (Fernández 1993). El sustrato va de limoso-arenoso a limoso, combinado con conchas de moluscos. Chametla presenta un oleaje mínimo y debido a la poca pendiente que tiene, la marea ejerce gran influencia sobre el área disponible, donde quedan expuestos durante la marea baja entre 500 y $1000 \mathrm{~m}$ lineales de humedal (Fernández 1993).

Para determinar la conducta alimentaria de las cuatro especies, se efectuaron visitas entre el 15 y el 22 de enero de 1993, durante las horas luz de cada día. Se hicieron observaciones de diferentes individuos de cada especie durante 15 min y en ambas mareas. Las observaciones se realizaron con un telescopio (1560X) a una distancia mínima de $50 \mathrm{~m}$ de los grupos de aves, para disminuir la perturbación.

La información se obtuvo según el método de Stenzel et al. (1976), se consideró éste ya que durante visitas anteriores, se observó que las especies de interés, seguían las estrategias descritas por dichos autores. Así, las estrategias utilizadas en la captura del alimento son: 1) Picoteo (Pk), cuando el ave picó una sola vez sobre la superficie del sustrato y levantó la cabeza. 2) Sondeo (Pb), cuando el ave hundió el pico en el sustrato y levantó la cabeza. 3) Picoteo múltiple (MPk), cuando el ave picó más de una vez sobre la superficie del sustrato levantando posteriormente la cabeza. 4) Sondeo múltiple (MPb), cuando el ave hundió el pico en el sustrato más de una vez sin levantar la cabeza. Además, se registró el éxito de captura (o fracaso): un intento exitoso se consideró cuando el ave realizó el movimiento requerido para tragar.

La búsqueda del alimento se consideró en función a los int/min; en cuanto al éxito bruto, éste se consideró como las presas/min. Es pertinente aclarar que un ave con altos presas/min puede obtenerlos por realizar un gran número de intentos o por tener una efectividad elevada, por lo que se utilizó un tercer índice, la eficiencia. Así, ésta dependerá de la relación que guarden los intentos y éxitos por minuto, dado que está definida como el cociente de los índices anteriores, expresada como porcentaje. Se planteó la hipótesis de que las frecuencias de int/min, presas/min y eficiencia para cada método dependen de la especie y del tipo de marea (viva: con lunas llena o nueva, muerta: con cuartos crecientes o menguante). Para corroborarlo se realizaron doce análisis de variancia de dos vías modelo II, ésto es cuatro métodos ( $\mathrm{Pk}, \mathrm{Pb}, \mathrm{MPk}$ y $\mathrm{MPb}$ ) por tres índices (int/min, presa/min y eficiencia), al $95 \%$ de confianza.

El tiempo total de observación fue de $20 \mathrm{~h}$ 3'. De las cuales 6h 1' le corresponden a L. fedoa, 4h 47'a N. phaeopus, 4h 37' a N. americanus y 4 h 38 ' a $C$. semipalmatus. A los diferentes grupos de datos (combinación especiemétodo-marea) se les aplicó la prueba de normalidad de Kolmogorov-Smirnov ( $\alpha=0.05)$. En todos los casos la hipótesis fue aceptada, el menor de los niveles de significancia fue del $12 \%$. También se realizaron pruebas de homocedasticidad de Barlett $(\alpha=0.05)$, en ellas se evidenció la igualdad de variancias, el menor nivel de significancia fue de $15 \%$. Los resultados anteriores permiten utilizar estadística paramétrica. Los resultados indican que el tipo de marea no afectó la frecuencia de utilización de los diferentes métodos (Cuadro 1). En contraste, la especie afecta a los int/min en todos los casos, disminuyendo su efecto para las presas/min; y prácticamente desapareciendo para la eficiencia (Cuadro 1). 


\section{CUADRO 1}

Nivel de significancia ( $p$ ) para el efecto de la especie (especie) y el tipo de marea (marea) para los 12 análisis de variancia realizados (cuatro métodos y tres índices alimentarios)

Tipo de método

\begin{tabular}{|c|c|c|c|c|c|c|}
\hline \multirow[t]{2}{*}{ Índice } & & $\mathrm{Pb}$ & $\mathrm{Pk}$ & $\mathrm{MPb}$ & MPk & $\mathrm{n}$ \\
\hline & especie & $0.038^{*}$ & $0.004^{*}$ & $0.043^{*}$ & $0.0006^{*}$ & 3,3 \\
\hline \multirow[t]{2}{*}{$\mathrm{int} / \mathrm{min}$} & marea & 0.5217 & 0.114 & 0.671 & 0.347 & 1,3 \\
\hline & especie & $0.0101^{*}$ & 0.072 & 0.052 & 0.398 & 3,3 \\
\hline \multirow[t]{2}{*}{ presa/min } & marea & 0.867 & 0.241 & 0.109 & 0.9542 & 1,3 \\
\hline & especie & 0.353 & 0.1001 & 0.468 & 0.364 & \\
\hline$($ presa / min) $* 100$ & marea & 0.768 & 0.854 & 0.833 & 0.625 & \\
\hline
\end{tabular}

*Significativamente diferentes.
Las aves se alimentaron con los cuatro métodos, preferentemente $\mathrm{Pk}$ y $\mathrm{Pb}$ (Cuadro 2). Pero se observaron diferencias en el número de int/min y en el método utilizado. Así, L. fedoa tuvo preferencia por Pk; además ésta utilizó más MPk y $\mathrm{MPb}$. N. phaeopus presentó un número menor de intentos, particularmente con MPk y MPb, predominando $\mathrm{Pk}$ y $\mathrm{Pb}$; aunque el primero fue más utilizado. N. americanus y C. semipalmatus utilizaron con mayor frecuencia Pk. Por otro lado, $N$. americanus fue la que más utilizó $\mathrm{Pb}$ (Cuadro 2).

En lo que se refiere a presas/min, L.fedoa fue la que capturó más presas; con $\mathrm{Pb}$ y MPb como los métodos más exitosos, sobre todo el primero. $N$. phaeopus fue la especie con menos presas/min con cualquier método, e incluso con MPk no capturó, Pk fue la técnica más empleada. $N$. americanus obtuvo más presas/min con Pb. Para C. semipalmatus, los cuatro métodos fueron exitosos, aunque se notó que prefiere $\mathrm{Pk}$ y $\mathrm{Pb}$ (Cuadro 2).

CUADRO 2

Promedio y desviación estándar () de los tres índices empleados (int./min, presas/min y efeciencia) para cada método de alimentación ( $P b, P k, M P b, M P k)$, por las cuatro especies de interés

\begin{tabular}{|c|c|c|c|c|c|}
\hline \multirow[b]{2}{*}{ Método } & \multirow[b]{2}{*}{ Índice } & \multicolumn{4}{|c|}{ Especies } \\
\hline & & L. fedoa & N. phaeopus & N. americanus & C. semipalmatus \\
\hline \multirow{2}{*}{$\mathrm{Pb}$} & $\mathrm{Int} / \mathrm{min}$ & $2.32(2.35)$ & $0.45(0.44)$ & $2.85(2.80)$ & $0.89(1.02)$ \\
\hline & Presas/min & $1.69(2.18)$ & $0.11(0.12)$ & $0.32(0.51)$ & $0.41(0.53)$ \\
\hline \multirow{4}{*}{$\mathrm{Pk}$} & Eficiencia & $55.85(32.69)$ & $23.33(26.94)$ & $12.45(14.20)$ & $37.31(39.57)$ \\
\hline & $\mathrm{Int} / \mathrm{min}$ & $3.29(2.40)$ & $0.79(0.75)$ & $4.12(2.32)$ & $4.14(2.85)$ \\
\hline & Presas/min & $0.18(0.17)$ & $0.15(0.18)$ & $0.17(0.24)$ & $0.49(0.84)$ \\
\hline & Eficiencia & $6.27(5.99)$ & $24.93(30.85)$ & $5.33(13.03)$ & $12.59(23.74)$ \\
\hline \multirow{3}{*}{$\mathrm{MPb}$} & $\mathrm{Int} / \mathrm{min}$ & $1.14(1.04)$ & $0.10(0.17)$ & $0.52(0.64)$ & $0.31(0.32)$ \\
\hline & Presas/min & $0.62(0.70)$ & $0.03(0.06)$ & $0.05(0.08)$ & $0.16(0.25)$ \\
\hline & Eficiencia & 41.32(28.66) & $11.90(20.89)$ & $10.02(18.26)$ & $25.52(44.95)$ \\
\hline \multirow{3}{*}{ MPk } & $\mathrm{Int} / \mathrm{min}$ & $1.18(0.92)$ & $0.04(0.10)$ & $0.24(0.28)$ & $0.64(0.75)$ \\
\hline & Presas/min & $0.12(0.17)$ & $0.00(0.00)$ & $0.01(0.04)$ & $0.14(0.26)$ \\
\hline & Eficiencia & $10.71(11.50)$ & $0.00(0.00)$ & $2.08(5.18)$ & $20.92(27.35)$ \\
\hline
\end{tabular}


$\mathrm{Pb}$ fue la técnica alimenticia que resultó ser más eficiente (Cuadro 2). De manera específica, L. fedoa tuvo el mayor porcentaje de eficiencia con $\mathrm{Pb}$ y $\mathrm{MPb}$; resaltando $\mathrm{Pb}$. En el caso de $N$. phaeopus a pesar de intentar alimentarse con $\mathrm{MPk}$, no obtuvo presas; y Pk y $\mathrm{Pb}$ fueron igual de eficientes (Cuadro 2). En el caso de $N$. americanus $\mathrm{Pb}$ y $\mathrm{MPb}$, fueron los métodos más eficaces (cuadro II). Para $C$. semipalmatus $\mathrm{Pb}$ fue el más eficaz aunque, para este playero los tres métodos restantes también fueron eficientes (Cuadro 2).

La marea (viva o muerta) tiene un bajo efecto en la frecuencia de utilización de los métodos, pues las aves responden antes con modificaciones en las áreas y/o tiempos de alimentación que con cambios en los métodos de captura (Turpie y Hockey 1993). Por otra parte, cada especie utiliza diferencialmente el o los métodos que en función de sus requerimientos y "habilidades" mejores resultados les dan (Wishart y Sealy 1980).

Es de resaltarse que a la eficiencia no la afecta ni la marea ni la especie. Al parecer, ésto debe ser interpretado en función a las diversas estrategias de alimentación. Es decir, algunas especies, en este estudio $N$. phaeopus y $N$. americanus, son más selectivas e invierten un considerable tiempo de manejo en presas de tallas grandes, que implican una mayor riqueza calórica. En contraste, especies como L. fedoa, son muy generalistas e invierten poco tiempo en la captura de presas pequeñas con bajos contenidos calóricos, pero compensan lo anterior con la captura de un alto número de éstas. Este efecto también es explicable, dadas las semejanzas en talla de las cuatro especies, pues es de esperarse que tengan requerimientos energéticos parecidos.

Las cuatro especies se están alimentando durante la mayor parte de las horas de luz del día. Lo que coincide con lo expuesto por Baker y Baker (1973), quienes mencionan que las aves migrantes invierten el mayor tiempo posible en la alimentación. Es probable que las cuatro especies se estén alimentando durante la noche, este comportamiento es común en aves con grandes demandas energéticas (e.g. vuelos largos, Robert y McNeil 1989). Este estudio se realizó a finales del período invernal, por lo que las aves estaban por iniciar la migración de primavera.

Ambas especies de Numenius se alimentaron depredando al cangrejo violinista ( $U c a$ crenulata). Al parecer, se reparten el recurso separándose en espacio (Kelly y Cogswell 1979, Brabata 1995), y utilizando técnicas diferentes ( $\mathrm{Pk} N$. phaeopus y $\mathrm{Pb} N$. americanus). $N$. phaeopus fue siempre más selectivo, ya que las presas/min del primero tuvieron un menor intervalo de variación con eficiencias mayores (Cuadro 2). Dada esta cualidad de "escoger", los depredadores deben poder cambiar de estrategia para responder a modificaciones bióticas y abióticas (Baker y Baker 1973). Así, podría explicarse el número similar de presas y eficiencia de $\mathrm{Pk}$ y $\mathrm{Pb}$ en ambas especies. $\mathrm{La}$ utilización de $\mathrm{Pk}$ se relaciona con la captura de presas grandes, es decir, estos depredadores invierten más tiempo y energía en la búsqueda y captura de alimento (Robert y McNeil 1989).

L. fedoa se alimentó siempre dentro del agua, lo cual coincide con las observaciones de Kelly y Cogswell (1979), Wishart y Sealy (1980) y Brabata (1995). Carmona (1997) encontró que la biomasa de posibles presas en la zona es considerablemente elevada durante el mes de enero, mismo en que se realizó este trabajo. Lo anterior explicaría los elevados valores de los índices manejados. Se considera que existe una relación directa entre el tamaño del ave y el de su presa, sin embargo, en Chametla L. fedoa se alimentó de presas pequeñas; al parecer ésta es una característica del género (Baker y Baker 1973, Baker 1977).

C. semipalmatus se distribuyó en todo el espacio disponible, pero buscó a sus presas preferentemente en la porción media de la zona intermareal. Stenzel et al. (1976) observaron el mismo tipo de distribución en Palo Alto, CA. C. semipalmatus se considera un playero oportunista (Stenzel et al. 1979), esta cualidad se refleja en que haya sido el único con eficiencias importantes con los cuatro métodos considerados.

En suma, L. fedoa es una especie generalista que basa su alimentación en presas pequeñas, 
por su parte si bien $C$. semipalmatus es también generalista captura presas mayores y en áreas diferentes; en cuanto a las dos especies de $\mathrm{Nu}$ menius, éstas son marcadamente especialistas y fundamentan su dieta en presas grandes que requieren manipuleos considerables.

\section{AGRADECIMIENTOS}

A J. Guzmán por su apoyo logístico, a G. Fernández por su ayuda en el diseño y trabajo de campo y a H. Reyes por sus críticas y sugerencias.

\section{REFERENCIAS}

Baker, M.C. \& A.E. Baker. 1973. Niche relationships among six species of shorebirds on their wintering and breeding ranges. Ecol. Monog. 43:193-212.

Baker, M.C. 1977. Shorebird food habits in the eastern canadian Arctic. Condor 79:56-62.

Brabata, G. 1995. Presencia y conducta alimenticia de cuatro especies de playeros (Scolopacidae) en la Ensenada de La Paz, B.C.S. Tesis de Licenciatura, Universidad Autónoma de Baja California Sur, La Paz, B.C.S., México.

Carmona, C. 1997. Relación entre la abundancia de aves playeras y organismos bentónicos en la playa "El Conchalito", Ensenada de La Paz, B.C.S., durante un ciclo anual. Tesis de Maestría, Centro Interdisciplinario de Ciencias Marinas, La Paz, B.C.S., México.
Espinoza, A. 1977. Los Principales Parámetros Físico-químicos de las Aguas de la Ensenada de La Paz, B.C.S. Informe de Labores Centro Investigaciones Biológicas de Baja California Sur.

Fernández, G. 1993. Importancia de la marisma de Chametla, Ensenada de La Paz, B.C.S., para la migración e invernación del playerito occidental (Calidris mauri) (Charadriiformes:Scolopacidae). Tesis de Licenciatura, Universidad Autónoma de Baja California Sur, México.

Kelly, P.R. \& H.L. Cogswell. 1979. Movements and habitat use by wintering populations of Willets and Marbled Godwits. Studies Avian Biol. 2:69-82.

Paulson, D. 1993. Shorebirds of the Pacific Northwest. University of British Columbia,. Vancouver, British Columbia. 406 p.

Robert, M. \& R. McNeil. 1989. Comparative day and night feeding strategies of shorebirds species in a tropical environment. Ibis 131:69-79.

Stenzel, L.E.; H.R. Huber \& G.W. Page. 1976. Feeding behavior and diet of the Long-billed Curlew and Willet. Wilson Bull. 88:314-332.

Turpie, J.K. \& P.A.R. Hockey. 1993. Comparative diurnal and nocturnal foraging behaviour and energy intake of premigratory Grey Plovers Pluvialis squatarola and Whimbrels Numenius phaeopus in South Africa. Ibis 135:156-165.

Wishart, R.A. \& S.G. Sealy. 1980. The summer time budget and feeding behaviour of Marbled Godwits ( $\mathrm{Li}$ mosa fedoa) in southern Manitoba. Can. J. Zool. 53:1277-1282. 